\title{
Clinical Practice Trends in Mechanical Ventilation for Acute Asthma
}

Asthma is a serious public health problem. It is estimated that $\sim 26$ million people have asthma, and it accounts for nearly 2 million emergency department visits per year. ${ }^{1}$ Asthma exacerbations are characterized by episodes of progressively worsening dyspnea, coughing, wheezing, and chest tightness or any combination of these symptoms. Increased air-flow obstruction and dynamic hyperinflation impair ventilatory efforts, potentially leading to respiratory muscle fatigue. Approximately $2-20 \%$ of ICU admissions are attributed to severe asthma, with intubation and mechanical ventilation being necessary in up to one third. ${ }^{2}$ There is a paucity of good-quality randomized controlled trials and meta-analyses dealing with the topic of mechanical ventilation in acute asthma. ${ }^{3,4}$

In this issue of RESPIRATORY CARE, the Milwaukee Initiative in Critical Care Outcomes Research (MICCOR) Group of Investigators present their data regarding the

See the Original Study on Page 644

utilization of mechanical ventilation for acute asthma by analyzing the Nationwide Inpatient Sample database. ${ }^{5}$ The Healthcare Cost and Utilization Project Nationwide Inpatient Sample is an annual, nationally representative, allpayer in-patient care database containing hospital discharge abstract data. It is a stratified sample of $\sim 20 \%$ of United States community hospitals. The strata include rural or urban location, number of beds, region, teaching status, and ownership. The database includes all discharges from each hospital. The authors analyzed the database to determine if use of mechanical ventilation and in-hospital mortality changed between 2000 and 2008. The authors report that the proportion of admissions for which invasive mechanical ventilation was used during the first 2 days decreased from $1.4 \%$ in 2000 to $0.73 \%$ in 2008 , whereas noninvasive ventilation (NIV) increased from 0.34 to $1.9 \%$.

The author has disclosed no conflicts of interest.

Correspondence: Saiprakash B Venkateshiah MD, Atlanta VA Medical Center, Suite 2A147, 1670 Clairmont Road, Decatur, GA 30033. E-mail: svenkateshiah@gmail.com.

DOI: $10.4187 /$ respcare. 03230
The significant increase in NIV is an interesting finding, and the exact reason behind this is unclear. Over the past decade, NIV has become the standard of care in the management of acute hypercapnic COPD and cardiogenic pulmonary edema, with a rich body of evidence supporting its use. One plausible explanation regarding the increased use of NIV is that increasing comfort and familiarity with NIV could have led clinicians to use NIV for nontraditional indications such as acute asthma and pneumonia. Another less likely explanation is that NIV might have been indiscriminately used in acute asthma without appreciating the paucity of evidence supporting its use for this indication. There was a significant decrease in the use of invasive mechanical ventilation during this time period. The authors also report that the adjusted mortality in acute asthma requiring NIV or invasive mechanical ventilation was unchanged from 2000 to 2008, as was the hospital stay.

We need to understand the advantages and limitations of interpreting data from administrative databases. Administrative data are an important source of information regarding health care delivery because they are readily available with minimal cost, and they give the researchers the ability to analyze data across multiple health care settings and many years. Another appealing aspect of the information obtained from administrative data is that it is representative of real-world practice patterns and involves unselected patient populations. Administrative data will continue to play a role in health sciences research given the absence of national clinical registries. However, readers should exercise caution before widespread acceptance of the results of the studies based on these data due to the inherent limitations of the administrative data. Administrative data are derived primarily from claims submitted by providers to receive payment. Hence, the primary and secondary International Classification of Diseases, 9th Revision, Clinical Modification (ICD-9-CM) code selections are often driven by reimbursement policies. There is wide variation in reimbursement for different diagnosis-related groups under prospective payment. This creates incentives to identify principal diagnoses associated with higher reimbursing groups. There may be an over-representation of well-reimbursed diagnostic codes or procedures and an under-representation of codes that are 
poorly or not reimbursed at all. There is also a possibility that the diagnostic codes may change over time, and heightened diagnostic awareness and technological advances may make it more likely that certain diagnoses are identified. This may affect the validity of analysis derived from such data and may lead to erroneous inferences. Another major limitation of administrative data is the absence of important prognostic indicators such as vital signs, test results, functional status, and medication administration information. Administrative data lack clinical detail, and there is no ability to track individual patients across time or care settings. Measures of complexity are limited to demographics and comorbid conditions as measured by secondary ICD-9-CM diagnosis codes. There is wide variability of documentation of secondary diagnoses among clinicians and over time. This can impact the relationship between comorbid conditions and mortality. Hence, it is always challenging to draw conclusions about mortality and the factors impacting mortality from these data. ${ }^{6,7}$

This study adds to the existing body of sparse literature about the trends in mechanical ventilation practices for treatment of acute asthma. Given the limitations of drawing inferences from administrative data, it is difficult to comment on hard end points such as mortality. Good-quality randomized controlled trials are the order of the day to help clinicians address such questions in the management of acute asthma, which is of immense public health importance. The jury is still out on the safety, utility, and efficacy of NIV for acute asthma.

Saiprakash B Venkateshiah MD Division of Pulmonary, Critical Care and Sleep Medicine Emory University School of Medicine Atlanta, Georgia and

Atlanta Department of Veterans Affairs Medical Center Decatur, Georgia

\section{REFERENCES}

1. United States Environmental Protection Agency. Asthma fact sheet. EPA-402-F-04-019. March 2013. http://www.epagov/asthma/pdfs/ asthma_fact_sheet_en.pdf. Accessed January 29, 2014.

2. McFadden ER Jr. Acute severe asthma. Am J Respir Crit Care Med 2003;168(7):740-759.

3. Brenner B, Corbridge T, Kazzi A. Intubation and mechanical ventilation of the asthmatic patient in respiratory failure. J Allergy Clin Immunol 2009;124(2 Suppl):S19-S28.

4. Nowak R, Corbridge T, Brenner B. Noninvasive ventilation. J Allergy Clin Immunol 2009;124(2 Suppl):S15-S18.

5. Nanchal R, Kumar G, Majumdar T, Taneja A, Patel J, Dagar G, et al. Utilization of mechanical ventilation for asthma exacerbations: analysis of a national database. Respir Care 2014;59(5):644-653.

6. Sarrazin MS, Rosenthal GE. Finding pure and simple truths with administrative data. JAMA 2012;307(13):1433-1435.

7. Steiner C, Elixhauser A, Schnaier J. The healthcare cost and utilization project: an overview. Eff Clin Pract 2002;5(3):143-151. 